\title{
Oscillatory wave fronts in chains of coupled nonlinear oscillators
}

\author{
A. Carpio* \\ Departamento de Matemática Aplicada, Universidad Complutense de Madrid, 28040 Madrid, Spain \\ L. L. Bonilla $* *$ \\ Departamento de Matemáticas, Universidad Carlos III de Madrid, Avda. Universidad 30, E-28911 Leganés, Spain
}

(Dated: February 2, 2008)

\begin{abstract}
Wave front pinning and propagation in damped chains of coupled oscillators are studied. There are two important thresholds for an applied constant stress $F$ : for $|F|<F_{c d}$ (dynamic Peierls stress), wave fronts fail to propagate, for $F_{c d}<|F|<F_{c s}$ stable static and moving wave fronts coexist, and for $|F|>F_{c s}$ (static Peierls stress) there are only stable moving wave fronts. For piecewise linear models, extending an exact method of Atkinson and Cabrera's to chains with damped dynamics corroborates this description. For smooth nonlinearities, an approximate analytical description is found by means of the active point theory. Generically for small or zero damping, stable wave front profiles are non-monotone and become wavy (oscillatory) in one of their tails.
\end{abstract}

PACS numbers: 45.05.+x; 05.45.-a; 83.60.Uv

\section{INTRODUCTION}

Wave fronts and pulses play important roles in many physical systems. Examples abound: the motion of dislocations [1, 2] or cracks [3] in crystalline materials, atoms adsorbed on a periodic substrate [4], the motion of electric field domains and domain walls in semiconductor superlattices [5, 6], pulse propagation through myelinated nerves 7], pulse propagation through cardiac cells [8], etc. Furthermore, these localized waves often play an important role in Statistical Mechanics [9] or Quantum Field Theory [10]. When wave fronts or pulses are solutions of spatially discrete systems, they often fail to propagate unless an external force or parameter surpasses a critical value [1]]. Wave front pinning in discrete systems may be related to such different physical phenomena as the existence of Peierls stresses in continuum mechanics 12 or the relocation of electric field domains in semiconductor superlattices 6$]$. In the continuum limit, the width of the pinning interval (range of the external force for which wave fronts fail to propagate) tends to zero exponentially fast and many authors have calculated the critical force for different models in this limit [2, 13, 14, 15].

Not surprisingly, wave front motion and pinning are different depending on the dynamics describing the model at hand. To be precise, let us consider a chain 
of nonlinear oscillators, diffusively coupled and subject to an external force $F$ which acts as a control parameter:

$$
m \frac{d^{2} u_{n}}{d \tau^{2}}+\frac{d u_{n}}{d \tau}=u_{n+1}-2 u_{n}+u_{n-1}-A g\left(u_{n}\right)+F
$$

Typical nonlinearities $g(u)=V^{\prime}(u)$ are cubic, such that $A g(u)-F$ has three zeros, $U_{1}(F / A)<U_{2}(F / A)<$ $U_{3}(F / A)$ in a certain force interval $\left(g^{\prime}\left(U_{i}(F / A)\right)>0\right.$ for $\left.i=1,3, g^{\prime}\left(U_{2}(F / A)\right)<0\right)$. Moreover, $g(u)$ is symmetric with respect to $U_{2}(0)$. Examples are the overdamped Frenkel-Kontorova $(\mathrm{FK}) \operatorname{model}(g=\sin u)[1]$ and the quartic double well potential $\left(V=\left(u^{2}-1\right)^{2} / 4\right)$ 14]. $A>0$ measures the strength of the coupling and $m$ the relative strength of inertial and friction terms. Wave front solutions $u_{n}=w(n-c \tau)$ join the two stable constant states $U_{1}(F / A)$ and $U_{3}(F / A)$ (or viceversa) as $n$ increases from $-\infty$ to $\infty$.

Consider the extreme cases of conservative $(m=\infty)$ and overdamped dynamics $(m=0)$. In the overdamped case, wave fronts generically either move if $|F|>F_{c}>0$ or are pinned if $|F| \leq F_{c}[11]$. The depinning transition at $F_{c}$ was described by Carpio and Bonilla [11] for large and moderate values of $A$, by King and Chapman [15] in the continuum limit $A \rightarrow 0$, and by Fáth [16] for a piecewise linear $g(u)$. In the conservative case and for generic cubic nonlinearities $g(u)$, there are two critical forces $F_{c d}$ and $F_{c s}$ with $0<F_{c d}<F_{c s}=F_{c}$. Wave fronts may propagate stably for $|F|>F_{c d}$ but there are stable stationary (pinned) wave fronts if $|F|<F_{c}$. Thus pinned and moving wave fronts may coexist if $F_{c d}<|F|<F_{c s}$. The values $F_{c s}$ and $F_{c d}$ correspond to the static and dy- namic Peierls stresses of the literature on dislocations [2]. Atkinson and Cabrera found exact expressions for the wave fronts corresponding to a piecewise linear $g(u)$ and calculated the relationship between $F$ and wave front velocity [17]. An approximate theory was found somewhat earlier by Weiner 18]. More recently, Schmidt [19] and later authors [20, 21] found exact monotone wave fronts of conservative or overdamped systems by constructing models with nonlinearities such that the desired wave fronts were solutions of the models. In particular, Flach et al 21] showed coexistence between moving and pinned monotone wave fronts of a discrete system with conservative dynamics and $F=0$. On the other hand, for a sine nonlinearity and $F=0$, the numerical computations of Peyrard and Kruskal [22] show that an initial profile close to the continuum sine Gordon soliton loses energy via emission of phonons and it becomes pinned after a sufficiently long time interval. They also found stable moving wave fronts for small positive $F$, consistent with our previous statement that, generically, $F_{c d}>0$.

In this paper, we study the wave fronts of the damped system (11) and the transitions at $F_{c d}$ and $F_{c s}$. In contrast with previous work, we find wave fronts that are non-monotone, presenting wavy tails at one or both sides of a transition region in which the profile $w(n-c \tau)$ jumps an amount close to $\left[U_{3}(F / A)-U_{1}(F / A)\right]$. We call them wavy wave fronts. These fronts with wavy profiles persist even in the conservative limit $(m \rightarrow \infty)$ [23], and in fact Atkinson and Cabrera's wave fronts are also wavy, 
as these authors would have found out had they depicted their exact expression graphically. In the overdamped limit $m \rightarrow 0, F_{c d} \rightarrow F_{c}$ and the wave front profiles become monotone. We have thus arrived to a general picture of wave fronts in discrete chains of coupled nonlinear oscillators with $m>0$.

The rest of the paper is organized as follows. Section II considers Eq. (11) with a piecewise linear $g(u)$. We find exact formulas for the wave front profiles in the general damped case following the method of Atkinson and Cabrera's [17]. These profiles are often wavy and they are asymptotically stable in the damped case. It is important to obtain them for two reasons: (i) there are very few exact wave front solutions that are non-monotone, and (ii) in the limit of large inertia, it is hard to discriminate numerically between wavy wave fronts traveling with different velocities or having different profiles. Exact solutions make good benchmarks for numerical methods. The results for the damped model with a generic cubic nonlinearity are presented in Section III We calculate the static and dynamic Peierls stresses for typical values of $A$ and $m$. A characterization of these stresses is given in terms of our active point theory. Sec. IV contains a discussion of our results.

\section{EXPLICIT CONSTRUCTION OF WAVE FRONT PROFILES}

Let us rescale time in Eq. (11), so that $t=\tau / \sqrt{m}$, and consider a piecewise linear $g(u)$ :

$$
\begin{array}{r}
\frac{d^{2} u_{n}}{d t^{2}}+\alpha \frac{d u_{n}}{d t}=u_{n+1}-2 u_{n}+u_{n-1}-A g\left(u_{n}\right)+F \\
g\left(u_{n}\right)= \begin{cases}u_{n}+1, & \text { for } u_{n}<0, \\
u_{n}-1, & \text { for } u_{n} \geq 0\end{cases}
\end{array}
$$

where $\alpha=1 / \sqrt{m}$. Notice that $g(u)=u+1-2 H(u)$, where $H(x)=1$ for $x>0$ and $H(x)=0$ for $x<0$ is the Heaviside unit step function. Let us consider a smooth wave front profile $u_{n}=w(x) \equiv v(x)-1, x=n-c t$, moving rigidly with velocity $c$. We center the wave front so as to have $w(0)=0$. Taking into account that $g(u)$ is an odd function and using the front profile $u_{n}(t)=$ $w(n-c t)$, we can see that the following transformations leave Eq. (2) for $w(x)$ invariant:

$$
\begin{gathered}
(x, w, c, F) \rightarrow(-x, w,-c, F) \\
(x, w, c, F) \rightarrow(x,-w, c,-F) \\
(x, w, c, F) \rightarrow(-x,-w,-c,-F) .
\end{gathered}
$$

Let us consider now the case $F>0, c>0$ and $w^{\prime}(0)<0$, i.e., a wave front profile that decreases in the transition region about $x=0$. The transformations (4) yield a profile with: (i) $c<0, F>0$ increasing in the transition region, (ii) $c>0, F<0$ increasing in the transition region, and (iii) $c<0, F<0$ decreasing in the transition region. Thus we find that $\operatorname{sgn} w=-\operatorname{sgn}(x c F), g(w)=$ $w+1-2 H(-x \operatorname{sgn}(c F))$, and we can restrict ourselves to considering the case $F>0, c>0$ and $w^{\prime}(0)<0$ : 

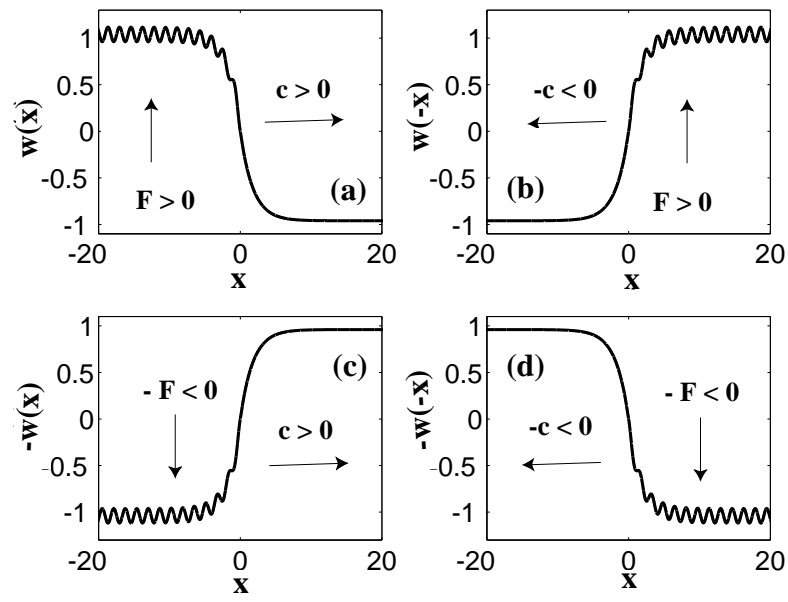

FIG. 1: Symmetries in the wavefront solutions for $A=0.25$, $\alpha=0, c=0.5$. and $F=0.009$.

All other three possible cases can be obtained from our results by using Eq. (4); see Figure 1

The wave front profile $v(x)=w(x)+1$ satisfies:

$$
\begin{array}{r}
c^{2} v^{\prime \prime}(x)-\alpha c v^{\prime}(x)-[v(x+1)-2 v(x)+v(x-1)] \\
+A v(x)=2 A H(-\operatorname{sgn}(c F) x)+F
\end{array}
$$

with $v(0)=1$. We can calculate $v(x)$ by using the contour integral expression for the step function:

$$
H(-x)=-\frac{1}{2 \pi i} \int_{C} \frac{e^{i k x}}{k} d k
$$

Here $C$ runs over the real axis in the complex $k$ plane passing above the pole at $k=0$ as in Fig. 2. For $x>0$ (resp. $x<0$ ), $C$ is closed by a semicircle in the upper (resp. lower) half plane oriented counterclockwise (resp. clockwise).

Then the solution of Eq. (5) is

$$
v(x)=\frac{F}{A}-\frac{A}{\pi i} \int_{C} \frac{\exp [i k \operatorname{sgn}(c F) x] d k}{k L(k, \alpha)},
$$

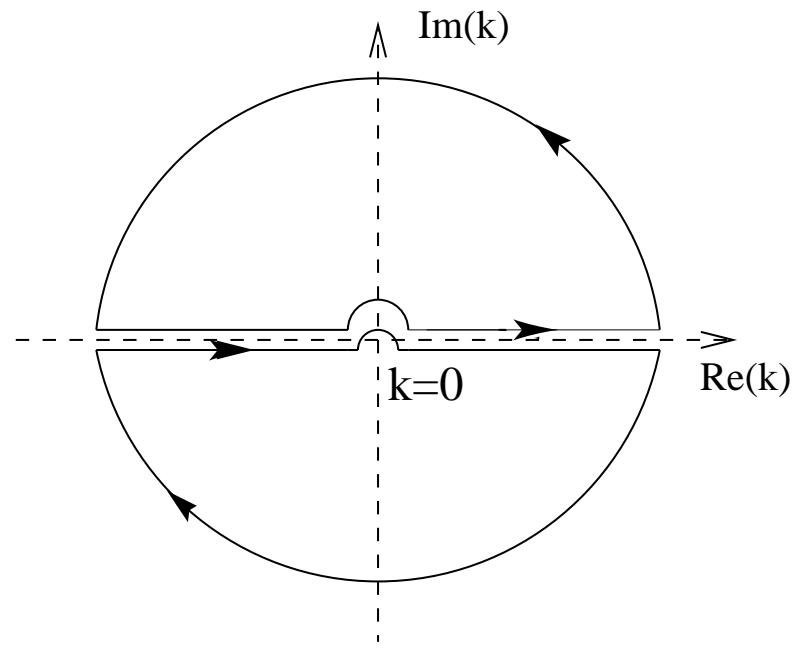

FIG. 2: Contour for the Heaviside step function (6) and the integral formula (7)- (8) when $\alpha \neq 0$.

$$
L(k, \alpha)=A+4 \sin ^{2}\left(\frac{k}{2}\right)-k^{2} c^{2}-i k|c| \alpha \operatorname{sgn}(F)
$$

All the zeros of the function $L(k, \alpha)$ given by (17) are complex for $\alpha>0$, and they correspond to exponentially localized modes. The nonzero poles of the integrand in Eq. (7) can be found graphically by plotting the curves $\operatorname{Re} L(k, \alpha)=0$ and $\operatorname{Im} L(k, \alpha)=0$ in the complex $k$-plane, as depicted in Fig [3] When $\alpha \rightarrow 0$, a finite number of poles tend to the real axis, whereas infinitely many keep a nonzero imaginary part even at $\alpha=0$. The poles on the real axis correspond to radiation modes, cause oscillations in the wave front tails, and their number increases as $c$ decreases; see Figs. 4(a) and (b). The purely imaginary poles of Figures 3 and 4 (c) yield the central monotone part of the wave front profiles. For $\alpha=0$, the integration contour in Eq. (7) avoids poles on the real axis according to a criterion due to Atkinson and Cabrera [17], shown in Figure 5] and derived later on this Section. 

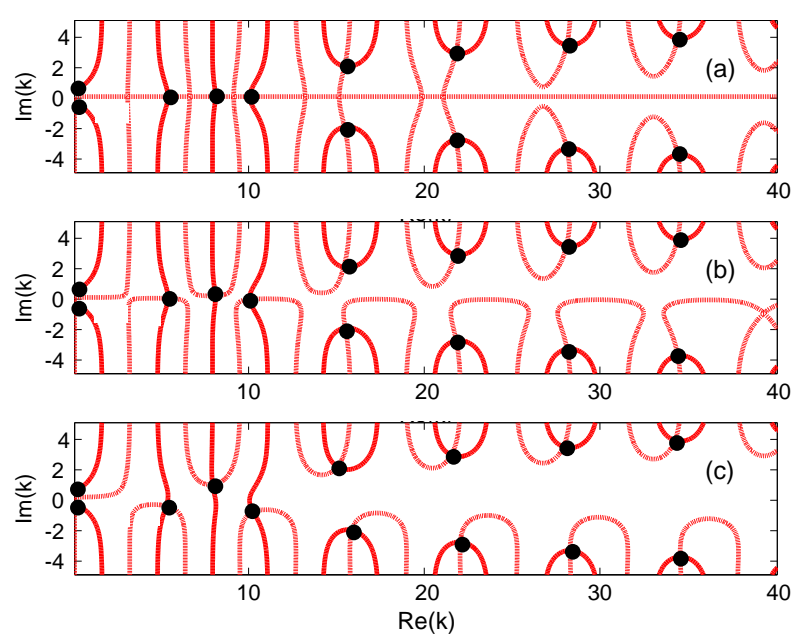

FIG. 3: Complex poles as intersection of the curves $\operatorname{Re} L(k, \alpha)=0$ and $\operatorname{Im} L(k, \alpha)=0$ when $c=0.2, A=0.25$ and: (a) $\alpha=0$, (b) $\alpha=0.1$, (c) $\alpha=1$.

We will use (7) and the method of residues to construct profiles satisfying $v(x)>1$ for $x<0$ and $v(x)<1$ for $x>0$. Notice that we can obtain a complex dispersion relation between $\omega=k c$ and $k$ from $L(k, \alpha)=0$. The contour choice and the fact that $\alpha>0$ give rise to an exponential decay of $v(x)$ to its asigned values at $x= \pm \infty$. When $\alpha=0$, the wave fronts may exhibit undamped oscillations extending all the way to infinity.

The condition $v(0)=1$ yields a relationship between the wave front velocity $c$ and the external force $F$ :

$$
\begin{aligned}
1 & =\frac{F}{A}-\frac{A}{\pi i} \int_{C} \frac{d k}{k L(k, \alpha)} \\
& =\frac{F}{A}-\sum_{L(p, \alpha)=0, \operatorname{Im}(p)>0} \frac{2 A}{p L_{k}(p, \alpha)},
\end{aligned}
$$

where we have assumed that $c F>0$ and $v^{\prime}(1)<0$. The resulting function $F(c)$ can be calculated by computing this series of residues numerically. Once $F(c)$ is known, Eq. (7) can be used to compute the wave front profiles for
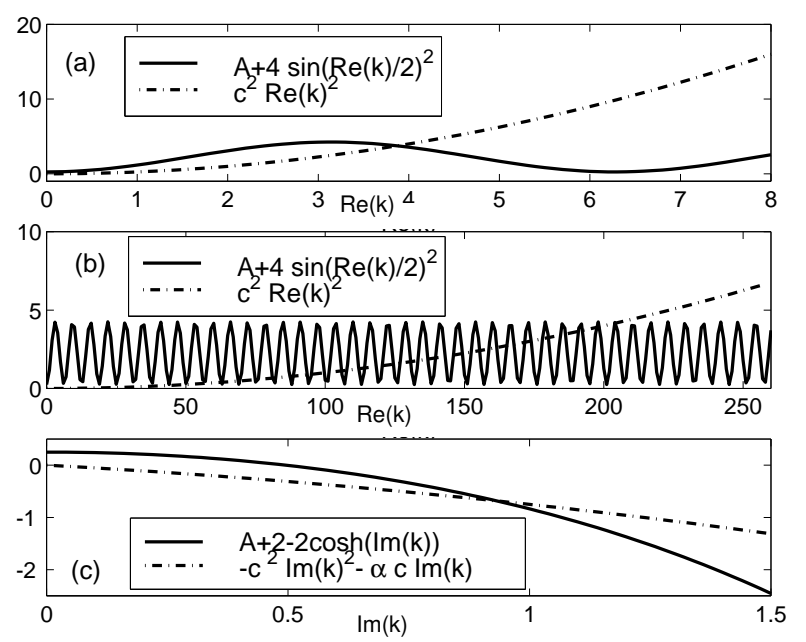

FIG. 4: Real poles in the conservative case $\alpha=0$ with $A=$ 0.25: (a) $c=0.5$ (b) $c=0.01$; Purely imaginary poles when $A=0.25$ (c) $c=0.5, \alpha=1$

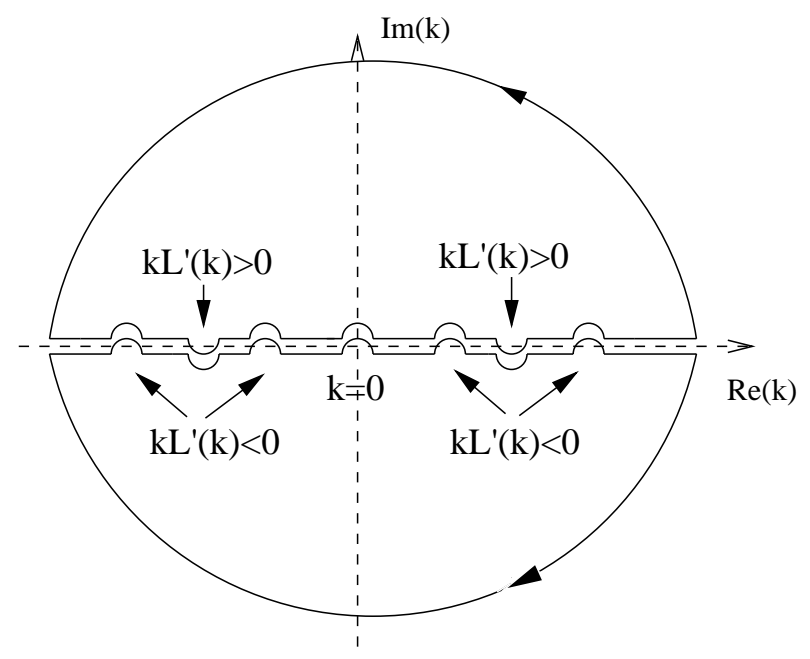

FIG. 5: Contour for the integral formula (7)-(8) in the conservative case $\alpha=0$ for $c>0$ and $F>0$.

a pair $(c, F(c))$. We shall now show how this construction works out for $\alpha=0, \alpha=\infty$ and for $\alpha$ finite. 


\section{A. Conservative case: $\alpha=0$}

It is instructive to see what happens in the conservative limit $\alpha \rightarrow 0+$. In this case, $L(k, 0)=0$ has real solutions, and we need a criterion to move the contour $C$ above or below the corresponding poles in the integral (7). To obtain it, we shall use the notation $L(k)=L(k, 0)$. Let $k_{0}$ be a real zero of $L(k)$. The complex zero of $L(k, \alpha)$ that becomes $k_{0}$ at $\alpha=0$ satisfies $0=L_{k}\left(k_{0}, 0\right)\left(k-k_{0}\right)+L_{\alpha}\left(k_{0}, 0\right) \alpha+\ldots$, which yields $k \sim k_{0}-L_{\alpha}\left(k_{0}, 0\right) \alpha / L_{k}\left(k_{0}, 0\right)$, that is,

$$
\begin{gathered}
k \sim k_{0}+\frac{i \alpha k_{0}|c| \operatorname{sgn} F}{L^{\prime}\left(k_{0}\right)} \Longrightarrow \\
\operatorname{sgn}(\operatorname{Im} k)=\operatorname{sgn}(c F) \operatorname{sgn}\left[c k_{0} L^{\prime}\left(k_{0}\right)\right],
\end{gathered}
$$

as $\alpha \rightarrow 0+$. We know that the contour $C$ in Eq. (6) lies in the upper (resp. lower) half plane provided $c F x>0$ (resp. $c F x<0$ ). Therefore, the poles whose residues count must satisfy $c F x \operatorname{Im} k>0$. Then Eq. (10) implies that we should count poles satisfying $x c k_{0} L^{\prime}\left(k_{0}\right)>0$. The physical meaning of this criterion becomes clear if we calculate the group velocity corresponding to mode $k_{0} . L(k)=0$ yields $(k c)^{2}=[\omega(k)]^{2}=A+4 \sin ^{2}(k / 2)$. Then $2 \omega(k) \omega^{\prime}(k)=4 \sin (k / 2) \cos (k / 2)=L^{\prime}(k)+2 k c^{2}$. Thus $v_{g} \equiv \omega^{\prime}(k)$ obeys

$$
v_{g}=c+\frac{L^{\prime}(k)}{2 k c} \Longrightarrow \operatorname{sgn}\left(v_{g}-c\right)=\operatorname{sgn}\left[c k L^{\prime}(k)\right]
$$

and we observe that the poles whose residues contribute to the solution satisfy $\left(v_{g}-c\right) x>0$. This was the criterion used by Atkinson and Cabrera [17]: All modes with $v_{g}>c$ must appear ahead of the wave front $(x>0)$, all those with $v_{g}<c$ must appear behind $(x<0)$. See Figure [5]

For $c>0$ and $\alpha=0$, the condition (9) becomes 17]

$$
\frac{F}{A}=\sum_{L(k)=0, k>0} \frac{2 A}{k\left|L^{\prime}(k)\right|}
$$

This formula follows straightforwardly from the fact that $L(k)$ and $k L^{\prime}(k)$ are even functions of (real) $k$ and symmetry considerations. Notice that our assumption $c>0$ has yielded $F>0$. The relation $F(c)$ given by (12) is plotted in Figure [6 for a value $A=0.25$ (see also Fig. 3 of Ref. 17). For a given value of the external force $F$, there may be several values of admissible velocities $c$, each corresponding to a different wave front profile. Thus different families of wave fronts (not all of them stable) may coexist for the same value of $F$. The function $F(c)$ presents different vertical asymptotes at positive values $c_{i}, c_{1}>c_{2}>\ldots$, where both $L(k)$ and $L^{\prime}(k)$ and vanish for positive $k$. We have $c_{n} \sim \sqrt{A} /(2 \pi n)$ as $n \rightarrow \infty$, so that the vertical asymptotes accumulate at $c=0$ as suggested by Fig. [4 In fact, the velocity can be eliminated from the two conditions $L(k)=L^{\prime}(k)=0$ yielding $A+2(1-\cos k)=k \sin k$. For large values of $k$, this gives $k \sim 2 \pi n+A /(2 \pi n)$, as the integer $n \rightarrow \infty$. The condition $L^{\prime}(k)=0$ then yields the previous formula for $c_{n}$.

The range of physical interest corresponds to $c_{1}<$ $c<1$ (wave front velocities larger than the largest resonant velocity but smaller than the sound speed 17] ). Let $\left(c_{m}, F\left(c_{m}\right)\right)$ be the minimum of the first and fastest branch of $F(c)$. Then $F_{c d}=F\left(c_{m}\right)$ yields the dynamic 

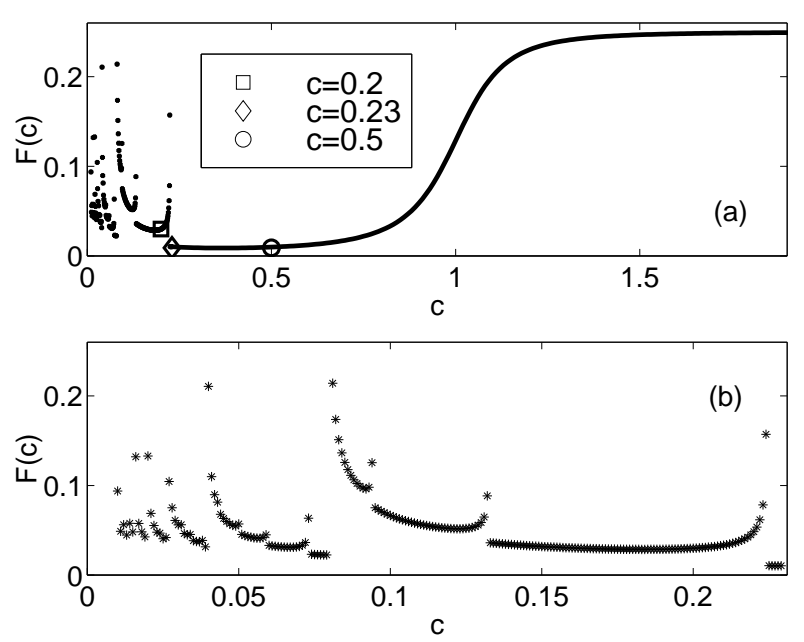

FIG. 6: (a) $F(c)$ for $\alpha=0, A=0.25$ as computed from formula (11), (b) Zoom in the region of resonances for $c$ small.
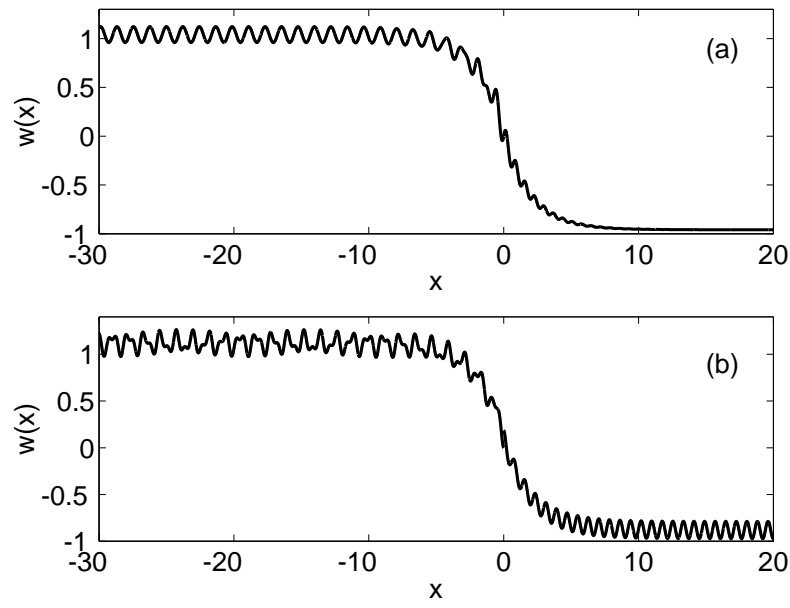

FIG. 7: Wave front profiles for $A=0.25, \alpha=0$ and: (a) $c=0.23,(\mathrm{~b}) c=0.2$.

Peierls force, under which the 'physical' branch of stable wave front profiles ceases to exist. This force is smaller than the static Peierls force, $F_{c}=A^{\frac{3}{2}}(A+4)^{-\frac{1}{2}}$, for the piecewise linear model [17].

Using the radiation condition $\left(v_{g}-c\right) x>0$, we have plotted in Figures 1 and 7 several wave front profiles. Fig- ure 1(a) shows a wave front profile for $\alpha=0, A=0.25$ and $F=0.009$. The wave velocity is $c=0.5$. The profile has been numerically approximated by computing the contour integral (7) as the series of residues truncated to a few terms. It is interesting to observe that the right tail of the wave front decays fast to $U_{1}(F / A)$ whereas the left tail oscillates about $U_{3}(F / A)$ with uniform amplitude. The reason for this behavior is that for the above parameter values, there are two real zeros of $L(k)$ at $\pm k_{0}$ with $k_{0} L^{\prime}\left(k_{0}\right)>0$ that contribute a non-decaying oscillation to the left tail. An infinitesimal amount of friction would dampen these oscillations by contributing a multiplicative factor $\exp \left\{-\left[\alpha k_{0} c / L^{\prime}\left(k_{0}\right)\right]\right\}$ to their amplitude. Figure [7(a) shows a wave front profile for $c=0.23$. Notice the decaying small oscillation in the right tail. We have still two real poles, but now $c=0.23$ is placed at the left of the minimum in the first branch of $F(c)$; see Figure 6(a). Figure Z(b) shows a wave front profile for $c=0.2$, in the second branch of $F(c)$, past the first resonance. Now we have three pairs of real poles. Two of them contribute to the oscillation in the left tail, the other one produces the oscillation in the right tail.

The dynamical stability of the constructed wave front solutions can be numerically checked by using their computed profile as initial data to solve Eq. (2) with $\alpha=0$ 24]. The results are compared at a fixed time $t=60$ to the expected configuration $w(n-c t)$ in Figure 8 The choice $c=0.5$ seems to produce a stable wave front. The choice $c=0.23$, (still on the first and fastest branch of 

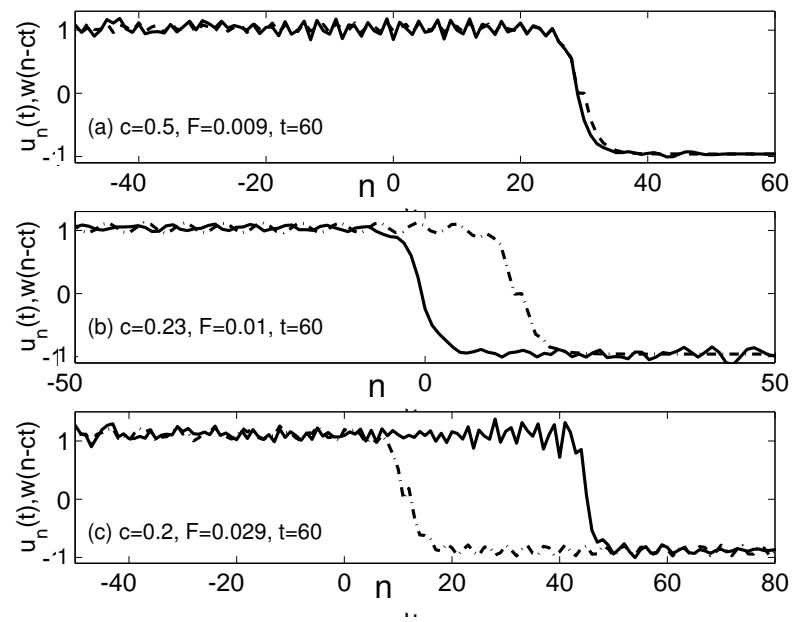

FIG. 8: Dynamical stability when $A=0.25, \alpha=0$. We compare $u_{n}(t)$ (solid line) to $w(n-c t)$ (dot-dashed line) for (a) $c=0.5$, (b) $c=0.23$, (c) $c=0.2$.

$F(c)$ but to the left of its minimum), evolves towards a static front. The choice $c=0.2$ (on the second branch of $F(c)$ ), evolves towards a wave front moving faster than expected, with a speed on the first branch of $F(c)$. Thus our numerical results seem to indicate that stable wave fronts have velocities on the first and fastest branch of $F(c)$ with $F^{\prime}(c)>0$, to the left of the minimum speed on this branch, $c_{m}>0$. Then $F_{c d}=F\left(c_{m}\right)$, and stable wave fronts with $v^{\prime}(1)<0$ have speeds larger or equal than $c_{m}$.

We have found wave front profiles with oscillatory tails that seem stable under small disturbances. One question that comes to mind is whether these profiles occur in models with smooth nonlinearities. The answer is yes: See an explicit construction in Appendix A.
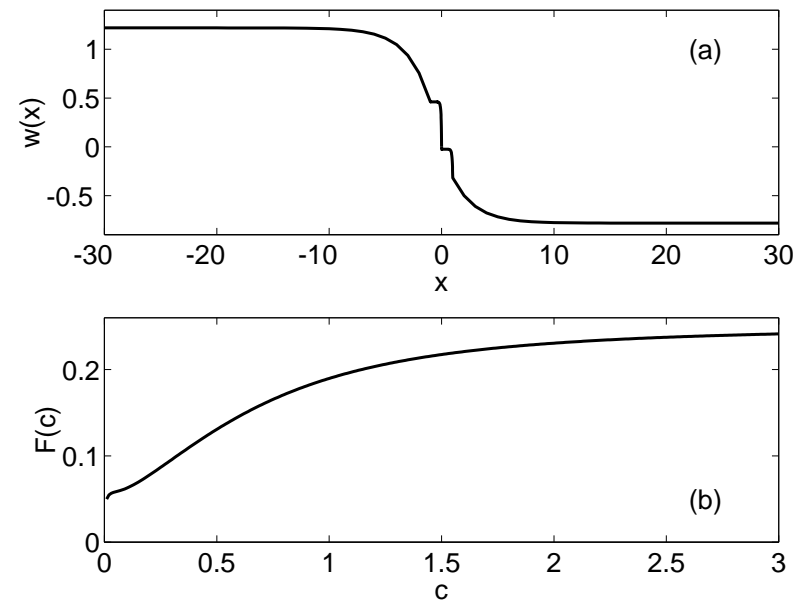

FIG. 9: Overdamped limit when $A=0.25$. (a) Wave front profile for $c=0.02$. (b) $F(c)$ as computed from formula (9).

\section{B. Overdamped limit: $\alpha=\infty$}

The results in the overdamped limit $m=0$ are consistent with previous work [11, 15, 16]: there are one wave front profile and one $c$ for each fixed $F$ above a threshold, $F_{c}$. Wave front profiles are monotone, and they resemble staircases for $c$ small. See Figure 9

\section{Finite damping: $\alpha>0$}

The results for finite damping interpolate between the conservative and overdamped cases. For small $\alpha$, the function $F(c)$ and the wave front profiles are non monotone although their oscillations decay as $n \rightarrow \pm \infty$; see Fig. 10 Fig. 11] shows a comparison between $u_{n}(t)$ for $\alpha=0$ (for the same values as in Fig. (8). We observe that, for this small damping, the corresponding wave fronts have the same stability properties as in the conservative case: dynamically stable for $c>c_{m}$, and unstable for 

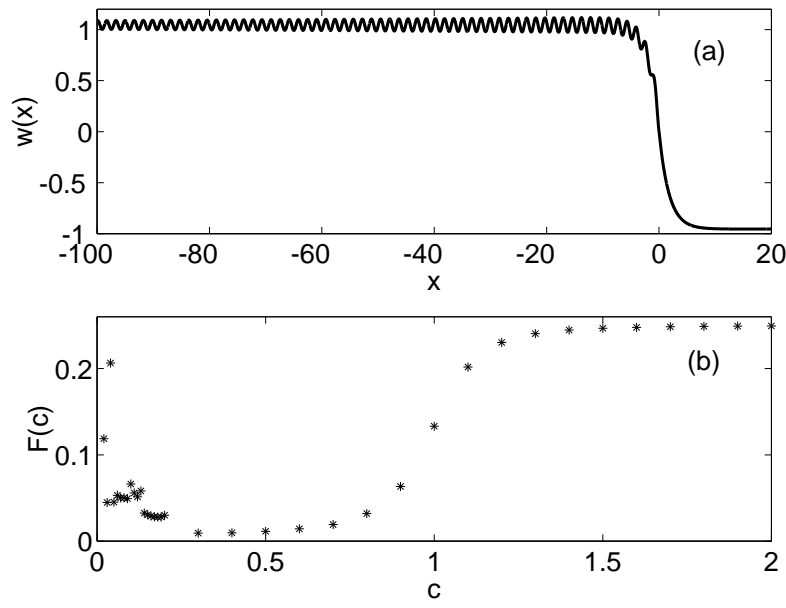

FIG. 10: Results for $A=0.25, \alpha=0.01$ (a) Wave front profile for $c=0.5$, (b) $F(c)$ as computed from formula (9).
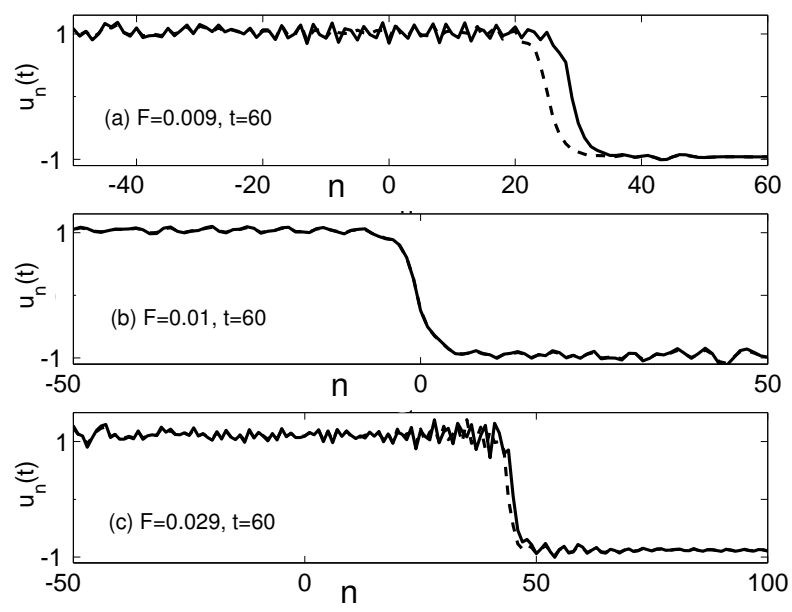

FIG. 11: Comparison between $u_{n}(t)$ calculated for $\alpha=0$ for the same values as in Fig. 8 (solid line) and $u_{n}(t)$ for $\alpha=0.01$ (dot-dashed line). (a) $F=0.009$, (b) $F=0.01$, (c) $F=0.029$.

$c<c_{m}$. Moreover there are dynamic and static Peierls stresses which are different from each other, as in the case $\alpha=0$.

It is interesting to ascertain the shape of the function $F(c)$ because its inverse function constitutes a bifurcation diagram clarifying wave front depinning. In the conser- vative case, $F(c)$ has infinitely many vertical asymptotes (resonances) accumulating at $c=0$. As $\alpha \rightarrow 0+$, the conditions $L(k, \alpha)=L_{k}(k, \alpha)=0$ yield

$$
\begin{aligned}
& k \sim k_{0}+\left|\frac{\alpha k_{0} c}{\cos k_{0}-c^{2}}\right|^{\frac{1}{2}} e^{i \frac{\pi}{4} \chi+i \pi l}, \quad l=0,1, \\
& \chi=\operatorname{sgn}\left[\left(\cos k_{0}-c^{2}\right) k_{0} F\right] .
\end{aligned}
$$

This formula shows how the double poles $k=k_{0}$ of the conservative case split when an infinitesimal friction is present. Then the vertical asymptotes of $F(c)$ at $c=c_{n}$ give rise to local maxima of $F(c)$ for small $\alpha>0$. These maxima are hard to resolve numerically (see Fig. 10(b)), but they can be approximately calculated as follows. Only poles with positive imaginary part contribute to the sum in Eq. (9). For these poles, the exponential factor in Eq. (13) is $(\chi+i) / \sqrt{2}$, and their contribution to the sum in Eq. (9) is approximately given by

$$
\frac{(2 \alpha)^{-\frac{1}{2}} A(\chi-i)}{k_{0} \operatorname{sgn}\left(\cos k_{0}-c^{2}\right)\left|k_{0} c\left(\cos k_{0}-c^{2}\right)\right|^{\frac{1}{2}}} .
$$

To this expression, we should add its complex conjugate, a contribution to the sum in Eq. (9) due to the pole $-k_{0}$. If we keep only these contributions in Eq. (9), thereby assuming that the considered maximum of $F(c)$ is large, $F_{M} \gg 1$, we obtain

$$
\left|F_{M}\right| \sim \frac{\sqrt{2} A^{2} \alpha^{-\frac{1}{2}}}{\left|k_{0}\right|^{\frac{3}{2}}\left|c\left(\cos k_{0}-c^{2}\right)\right|^{\frac{1}{2}}} .
$$

Now, in the conservative case, $c_{n} \sim \sqrt{A} /(2 \pi n)$ and $k_{0} \sim$ $2 \pi n+A /(2 \pi n)$ as the integer $n \rightarrow \infty$. Then the right side of Eq. (15) becomes proportional to $c_{n}$. When $n$ is so large that $c_{n}$ is no longer large compared to $\sqrt{\alpha}$, other terms of the sum contribute appreciably to $F$ in 
the formula (9). We conjecture that these contributions add to $F_{c s}$,

$$
\left|F_{M}\right|-F_{c s} \sim \sqrt{\frac{2}{\alpha}} A^{\frac{5}{4}} c_{n}, c_{n} \sim \frac{\sqrt{A}}{2 \pi n},
$$

so that the maxima of $F(c)$ accumulate near $c=0$ as the integer $n \rightarrow \infty$. We have depicted schematically the resulting $F(c)$ and the bifurcation diagram of $c$ versus $F$ in Fig. 12 Corresponding to the infinitely many local extrema in Fig $12($ a), there are infinitely many limit points (saddle-node bifurcations) in Fig. 12(b). Our numerical results indicate that only the branch of wave fronts with larger velocities in the physical interval $c_{m}<c$ and $|F|>F_{c d}$ are stable. This can be understood from the factorization theorems in Ref 25 . According to these theorems, one eigenvalue of the linear stability problem corresponding to the solution branches in the bifurcation diagram changes sign at limit points. If we use that the branch of wave fronts with larger velocity is stable, the branch that coalesces with it at the limit point $F=F_{c d}$ is unstable. This branch coalesces with another one at another limit point with larger $F$, and, there, a different eigenvalue of the linear stability problem changes sign from negative to positive. If this is so, the new branch is also unstable and all other wave front solution branches in Fig. 12(b) could also be unstable.

For larger values of $\alpha$, the wave front profiles become monotone, the oscillation amplitudes in $F(c)$ decrease and become difficult to appreciate; see Fig. 13] The transition from one parameter range to the other one occurs when the contribution from poles with small imaginary
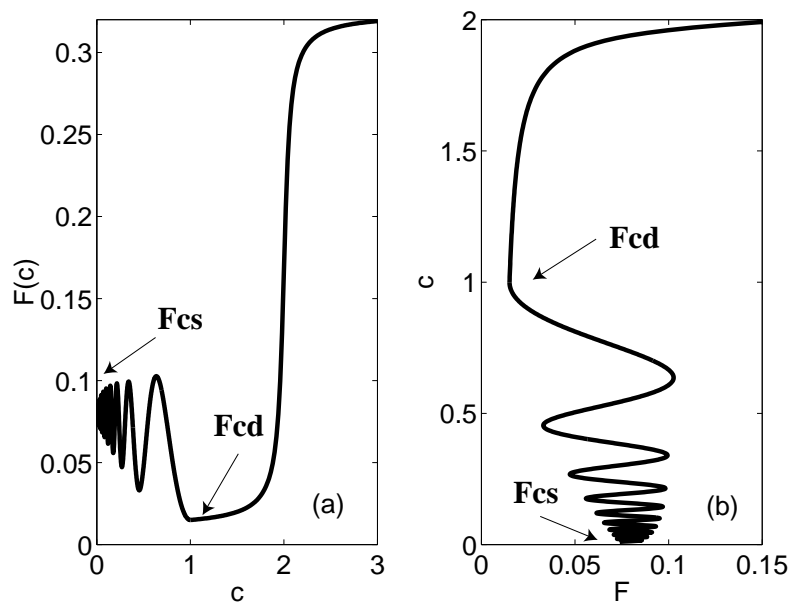

FIG. 12: (a) Schematic function $F(c)$ for small $\alpha>0$ showing infinitely many maxima accumulating at $c=0$ and $F=F_{c s}$. (b) The bifurcation diagram of wave front velocity versus $F$ : there are infinitely many limit points (saddle-node bifurcations) corresponding to the extrema of $F(c)$ in the interval $F_{c d}<F<F_{c s}$.
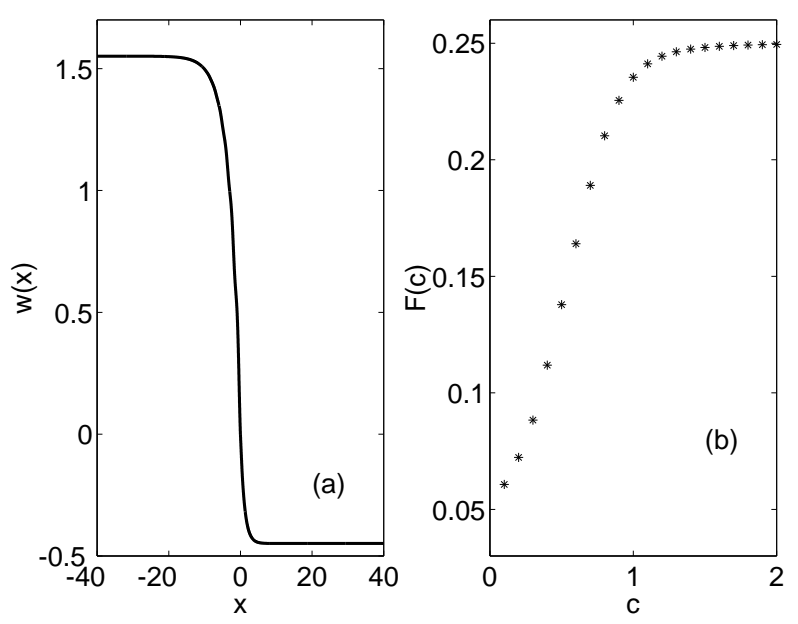

FIG. 13: Results for $A=0.25, \alpha=1$. (a) Wave front profile for $c=0.5$, (b) $F(c)$ as computed from formula (9).

part in Eq. (9) becomes relevant. 

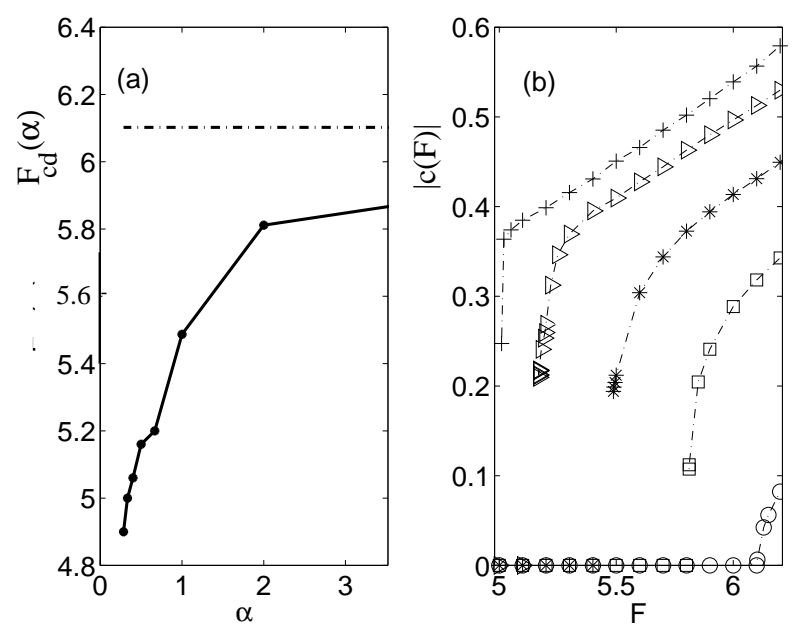

FIG. 14: Results for the Frenkel-Kontorova model with $A=$

10: (a) Dynamic (solid line) versus static (dot-dashed line) thresholds as functions of $\alpha$; (b) numerical velocities as functions of $F$ for decreasing values of $\alpha: 3$ (circles), 1.5 (squares), 1 (asterisks), 0.7 (triangles), 0.57 (crosses).

\section{WAVY WAVE FRONTS FOR GENERIC CUBIC NONLINEARITIES}

For generic smooth cubic nonlinearities $g(u)$, we cannot construct the wave front profiles by using contour integrals. However, we can extend our previous theory of the active points 11] for threshold phenomena to the case of finite damping. Thus we shall present numerical results for (relatively) large $A$ showing that wave fronts are similar to those for piecewise linear $g(u)$. Near $F_{c}$, we shall use the theory of active points to interpret numerical results.
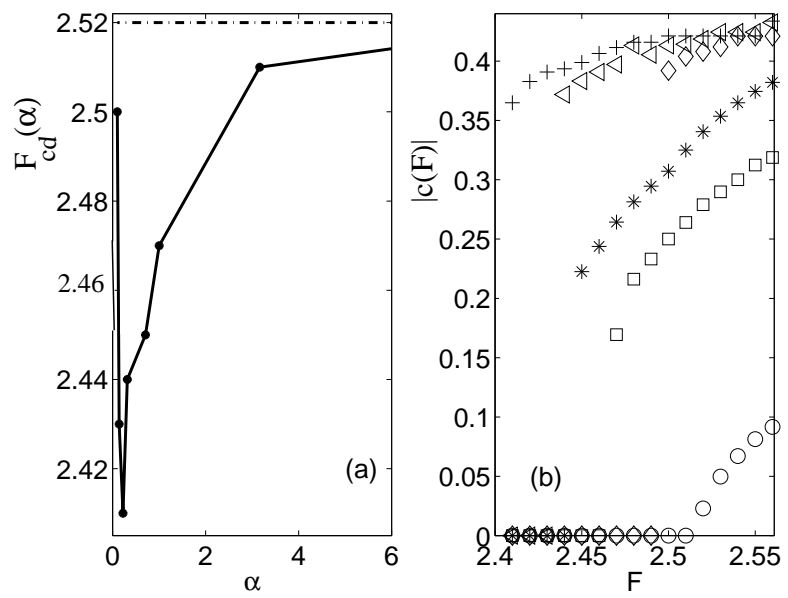

FIG. 15: Same as Fig. 14 for the model with a quartic potential. In (b), the values of $\alpha$ are: 3 (circles), 1 (squares), 0.7 (asterisks), 0.3 (triangles), 0.2 (crosses), 0.1 (diamonds).

\section{A. Numerical results}

Figures 14 and 15 show the dynamic and static Peierls stresses and the wave front velocity for the damped FK and cubic models, respectively. We have constructed the traveling wave fronts by solving numerically Eq. (2) in large lattices. For $F>F_{c s}$, we choose as initial data a static step-like profile: $u_{n}(0)=U^{(1)}(F / A)$ if $n \geq 0$, $u_{n}(0)=U^{(3)}(F / A)$ if $n<0, u_{n}^{\prime}(0)=0 \forall n$. We use the stable zeros $U^{(1)}(F / A)$ and $U^{(3)}(F / A)$ as boundary conditions for large $|n|$. The numerical solution $u_{n}(t)$ evolves very fast to a traveling wave $u_{n}(t)=w(n-c t)$ with a fixed constant value for the speed $c$. For $F$ below the static threshold, we choose as initial data the traveling solutions already found. As boundary condition, we use again $U^{(1)}(F / A)$ and $U^{(3)}(F / A)$. The numerical solutions $u_{n}(t)$ evolve to a traveling wave $u_{n}(t)=w(n-c t)$ 
with a profile and speed adjusted to the new value of $F$, provided $F$ is larger than the dynamical threshold $F_{c d}$. Below that value, the waves are pinned. The behavior of $c$ near $F_{c d}$ can be guessed from the known fact that the function $F(c)$ has a minimum $F_{c d}$ on its fastest branch at $c=c_{m}$ for piecewise linear $g(u)$; see Fig. [6] Near this minimum, $F \sim F_{c d}+\gamma\left(c-c_{m}\right)^{2}$ with $\gamma>0$, as indicated in Fig. [16(a). This yields $\left(c-c_{m}\right) \sim\left[\left(F-F_{c d}\right) / \gamma\right]^{\frac{1}{2}}$, a scaling that can be seen in Figs. 16(b) and (c), corresponding to smooth $g(u)$. The number $\gamma$ can be fitted by taking careful numerical measurements near $F_{c d}$. This seems to provide a good fitting over an interval of stresses that increases as $\alpha$ decreases. For larger friction values, taking values of $F$ farther from $F_{c d}$ produces a better fit to a scaling with the same exponent $1 / 2$ but with different $\gamma$; see the squares in Figs. 16(b) and (c).

Our numerical measurements of the speeds near $F_{c d}$ seem to indicate that (except in the overdamped limit $m=0)$ there is a critical non zero speed $c_{m}>0$ below which front propagation cannot be sustained. In the coexistence region, $F_{c d}<F<F_{c s}$, shown in Figs. 14 and 15. both the traveling wave fronts and the static wave fronts are dynamically stable. The wave front profiles for different damping values and the cubic $g(u)$ are depicted in Fig. 17 These profiles oscillate more and more as the damping coefficient decreases. For sufficiently large $\alpha$, the wave front profiles are monotone and become similar to those calculated in the overdamped limit $[11] . F_{c d}$ and $F_{c s}$ are almost equal. Similarly, as $A$ decreases and
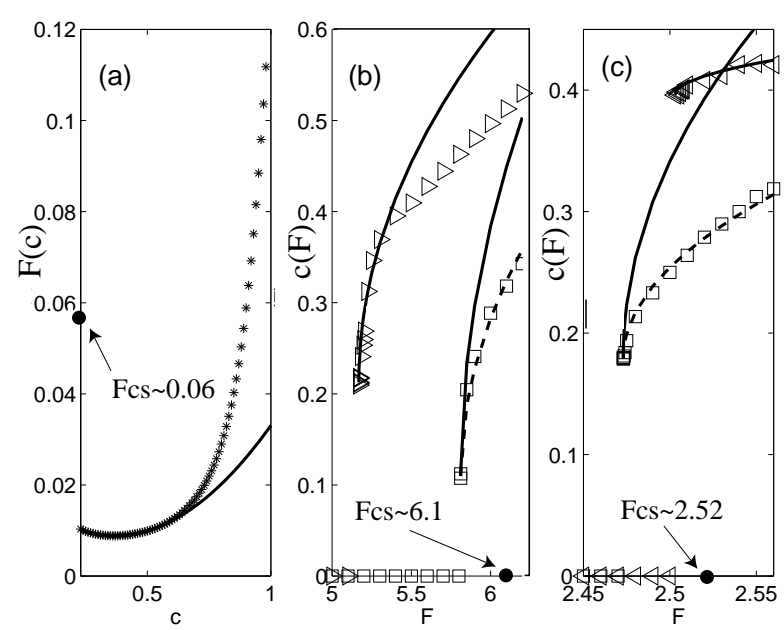

FIG. 16: (a) Function $F(c)$ for the piecewise linear $g(u)$ with $A=0.25$ and $\alpha=0$. (b) Velocity versus applied stress for $g(u)=\sin u$ with $A=10$ and $\alpha=1.4$ (squares) and $\alpha=$ 0.57 (triangles). (c) Velocity versus applied stress for $g(u)=$ $u\left(u^{2}-1\right)$ with $A=10$ and $\alpha=1.0$ (squares) and $\alpha=0.1$ (triangles).

we approach the continuum limit, the gap between static and dynamical thresholds is difficult to appreciate.

There is an important difference between models with a periodic nonlinearity such as FK and models with a cubic $g(u)$. In both cases, wave fronts can be constructed numerically for sufficiently large values of the damping. For $A=10$, wave fronts of the cubic model can be numerically found at least for $\alpha \geq 0.08$, whereas those of the FK model are found easily for larger damping, $\alpha>0.5$. For smaller values of the damping, the amplitude of the wave front oscillatory tails becomes so large that the FK wave front profile tends to jump between different periods of the nonlinearity: moving staircases are thus generated. 

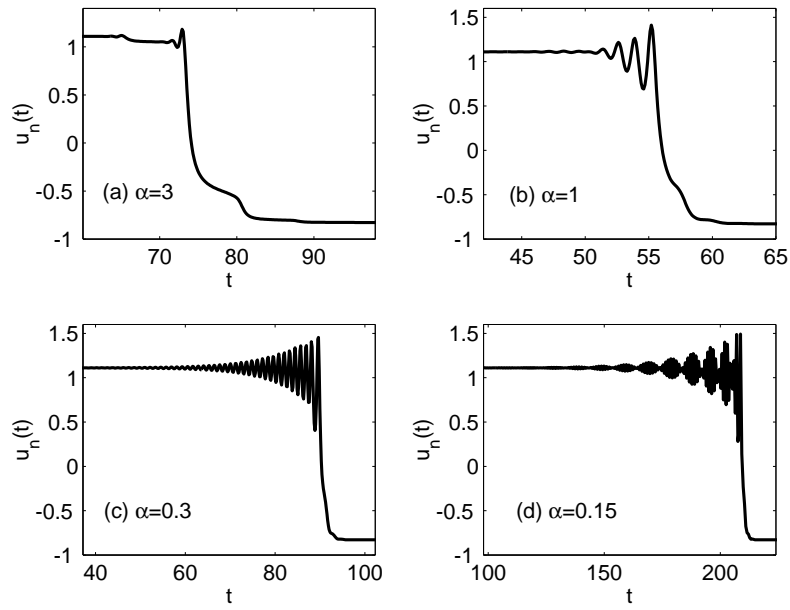

FIG. 17: Wave front profiles for the quartic potential as seen from the trajectory of a single point $u_{n}(t)$ : (a) $\alpha=3$, (b) $\alpha=1.5$, (c) $\alpha=0.3$, (d) $\alpha=0.15$.

\section{B. Active point theory}

To get approximate formulas for the wave front profile and velocity in the strongly discrete limit, $A \gg 1$, we can resort to the active point theory 11]. In this limit, there is one active point, say $u_{0}(t)$, and all others obey either $u_{n} \sim U_{1}(F / A)$ (for $\left.n>0\right)$ or $u_{n} \sim U_{3}(F / A)$ (for $n<0)$. We assume that the wave front we will construct has $F>0, c \geq 0$ and $w^{\prime}(0)<0$, as in the previous Section. According to Eq. (2), the active point satisfies the approximate equation:

$$
\begin{aligned}
\frac{d^{2} u_{0}}{d t^{2}}+\alpha \frac{d u_{0}}{d t} \approx & U_{1}\left(\frac{F}{A}\right)+U_{3}\left(\frac{F}{A}\right) \\
& -2 u_{0}-A g\left(u_{0}\right)+F
\end{aligned}
$$

This equation has three stationary solutions for $F<F_{c s}$, $z_{1}\left(F_{c} / A\right)<z_{2}\left(F_{c} / A\right)<z_{3}\left(F_{c} / A\right),\left(z_{1}\right.$ and $z_{3}$ are stable and $z_{2}$ is unstable), and only one stable stationary solution, $z_{3}$, for $F>F_{c s}$. The critical field $F_{c}=F_{c s}$ is such that the expansion of the right hand side of (17) about the two coalescing stationary solutions has zero linear term, $2+A g^{\prime}\left(u_{0}\right)=0$, and

$$
2 u_{0}+A g\left(u_{0}\right) \sim U_{1}\left(\frac{F_{c}}{A}\right)+U_{3}\left(\frac{F_{c}}{A}\right)+F_{c} .
$$

For $F$ sligthly above $F_{c}, u_{0}(t)=u_{0}\left(A, F_{c}\right)+v_{0}(t)$ obeys the following equation:

$$
\begin{aligned}
\frac{d^{2} v_{0}}{d t^{2}} & +\alpha \frac{d v_{0}}{d t} \approx a\left(F-F_{c}\right)+b v_{0}^{2} \\
a & =1+\frac{1}{A g^{\prime}\left(U_{1}\left(F_{c} / A\right)\right)}+\frac{1}{A g^{\prime}\left(U_{3}\left(F_{c} / A\right)\right)}>0 \\
b & =-\frac{A}{2} g^{\prime \prime}\left(u_{0}\right)>0
\end{aligned}
$$

where we have used $2+A g^{\prime}\left(u_{0}\right)=0$, 18) and ignored higher order terms. This equation has two distinguished limits, $\alpha \ll\left(F-F_{c}\right)^{\frac{1}{4}} \ll 1$ and $\alpha \gg\left(F-F_{c}\right)^{\frac{1}{4}}$. In the latter case, we can ignore the inertia in Eq. (19). The resulting reduced equation is exactly that analyzed in Ref. 11 except for a trivial rescaling of the time. The solution of that equation blows up at times $\left(t-t_{0}\right) \sim$ $\pm \pi \alpha /\left[2 \sqrt{a b\left(F-F_{c}\right)}\right]\left(t_{0}\right.$ is an arbitrary constant). Then the wave front velocity is approximately given by the reciprocal of the interval between two consecutive blow up times, namely,

$$
c=\frac{\sqrt{a b\left(F-F_{c}\right)}}{\pi \alpha} .
$$

After blow up, the wave front profile is reconstructed by inserting an inner layer, in which $u_{0}(t)$ obeys Eq. (17) with $F=F_{c}$, and it jumps from a neighborhood of $z_{1}\left(F_{c} / A\right)$ to $z_{3}\left(F_{c} / A\right)[11]$.

If $\alpha \ll\left(F-F_{c}\right)^{\frac{1}{4}} \ll 1$, we can ignore friction in Eq. (19) thereby obtaining a conservative dynamical system, 
Eq. (19) with $\alpha=0$, as our reduced equation. Its trajectories also blow up and the wave front velocity can be straightforwardly calculated as

$$
c=\frac{\left(\frac{a b\left(F-F_{c}\right)}{3}\right)^{\frac{1}{4}}}{2 \sqrt{2} K\left(\frac{1}{\sqrt{2}}\right)}=\frac{\sqrt{2 \pi}\left(\frac{a b\left(F-F_{c}\right)}{3}\right)^{\frac{1}{4}}}{\left[\Gamma\left(\frac{1}{4}\right)\right]^{2}}
$$

where $K(1 / \sqrt{2})=1.854075$ is the complete elliptic integral of the first kind with module $k=1 / \sqrt{2}$ 26]. Contrary to the overdamped case, a consistent inner layer connecting blowing up trajectories of the reduced equation and trajectories of Eq. (17) for $F=F_{c}$ and $\alpha=0$ does not exist. This points out to a breakdown of the active point theory as $\alpha \rightarrow 0+$, which is consistent with our conjectured bifurcation diagram in Fig. 12 (b) for the piecewise linear model. Assuming that Fig. 12(b) is also the bifurcation diagram of models with smooth nonlinearities, a succession of infinitely many saddle-node bifurcations (that accumulate at $c=0, F=F_{c s}$ ) connect the branch of stable wave fronts and that of stationary front solutions between $F_{c d}$ and $F_{c s}$. If this is the case, seeking a description in terms of standard normal forms as (19) and scaling is rather hopeless. Not surprisingly, the suspicious scaling (23) is hard to check numerically (the stable branch of moving wave fronts ends at $F=F_{c d}<F_{c}$ ), whereas the scaling (22) can be easily checked $\left(F_{c d} \approx F_{c}\right.$ in the overdamped limit) 11].

Let us now come back to Eq. (17) to explain the coexistence of moving and stationary wave fronts for $F_{c d}<F<F_{c}$. In Fig. [18 we have depicted the phase plane associated to Eq. (17) for the cubic non-

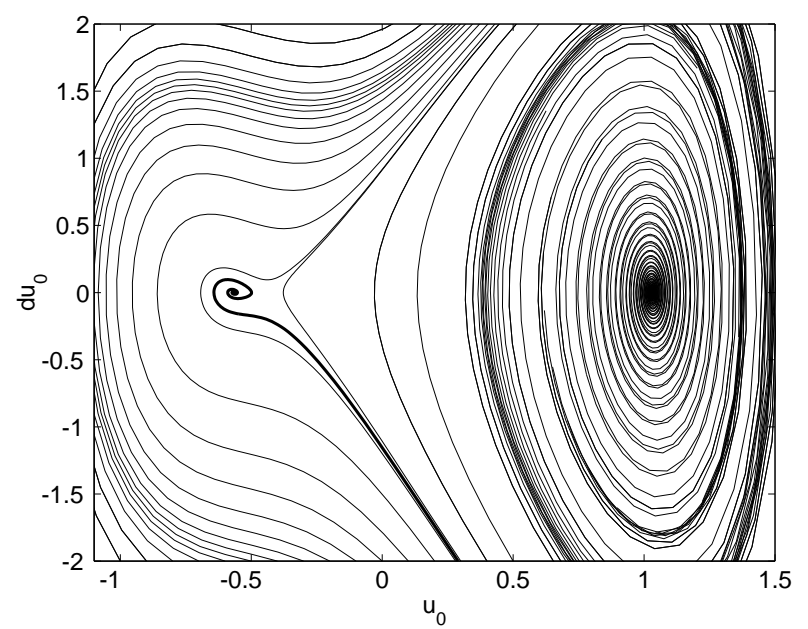

FIG. 18: Phase plane of Eq. (17) with the cubic nonlinearity for $A=10, \alpha=0.5$ and $F=2.45$.
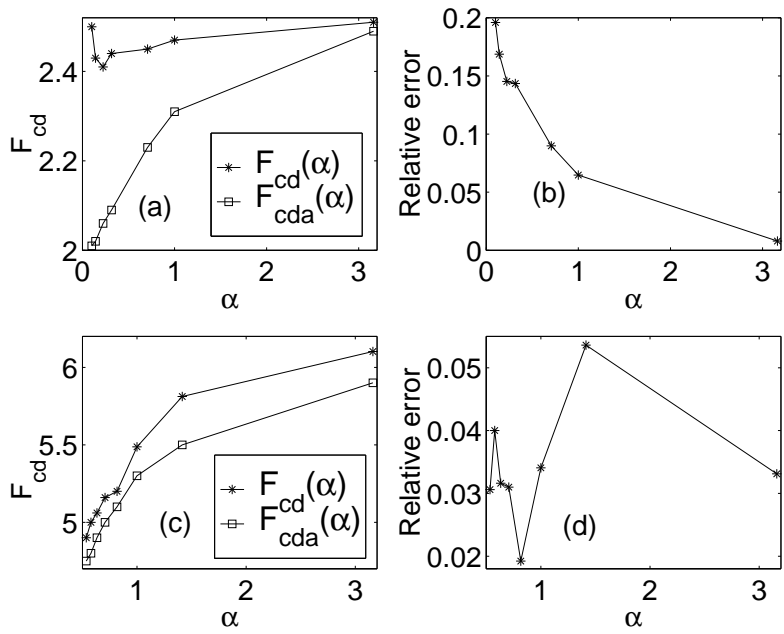

FIG. 19: Comparison of $F_{c d a} \equiv F_{c 2}$ and $F_{c d}$ as functions of the damping $\alpha$ for $A=10$ and: (a) the cubic nonlinearity, (b) its relative error, (c) the FK nonlinearity, and (d) its relative error.

linearity $g(u)=u\left(u^{2}-1\right)$. The profiles in Fig. 17 correspond to one active point jumping from a neighborhood of $U_{1}(F / A)$ to a neighborhood of $U_{3}(F / A)$. The right side of Eq. (17) has three zeros in the interval $\left[U_{1}(F / A), U_{3}(F / A)\right]$ for $F<F_{c_{1}}$, corresponding to 
two stable spiral points or centers $\left(z_{i}(F / A), 0\right), i=1,3$, and one saddle point $\left(z_{2}(F / A), 0\right)$. At $F=F_{c_{1}}$ two of the zeros coalesce and for $F>F_{c_{1}}$ only the spiral point $\left(z_{3}(F / A), 0\right)$ remains. For $\alpha>0$, there is a new critical value of $F, F_{c_{2}}$, such that the initial datum $\left(U_{1}(F / A), 0\right)$, which is close to the spiral point $\left(z_{1}(F / A), 0\right)$, may evolve to the other stable spiral point, $\left(z_{3}(F / A), 0\right)$, for $F \in\left(F_{c_{2}}, F_{c_{1}}\right)$; see Fig. 18 The trajectory leaving a neighborhood of $\left(z_{1}(F / A), 0\right)$ and entering $\left(z_{3}(F / A), 0\right)$ defines the wave front profile. For $F<F_{c 2}$, the initial datum $\left(U_{1}(F / A), 0\right)$ evolves toward $\left(z_{1}(F / A), 0\right)$ and no wave front is generated. Thus $F_{c 2}$ yields an approximation to the dynamical Peierls stress, $F_{c d}$. Fig. 19] compares $F_{c 2}$ to $F_{c d}$, which has been calculated numerically by solving the complete system (2). Notice that our approximation worsens as $\alpha$ decreases towards zero indicating break down of the one active point approximation. We shall explain below why this is so.

The dynamic critical Peierls stress can be intuitively explained as follows. The potential energy associated to the nonlinearity $h(u)=2 u_{0}+A g\left(u_{0}\right)-F-U_{1}(F / A)-$ $U_{3}(F / A)$ on the right side of Eq. (17) is depicted in Fig. 20(a). An initial condition $\left(U_{1}(F / A), 0\right)$ is close to the left minimum of $W\left(u_{0}\right)$. If the energy corresponding to the initial condition is slightly higher than that of the left minimum, the solution of (17) evolves toward it because of friction. On the other hand, larger energies cannot be dissipated by the friction term and the trajectory surpasses the maximum of $W\left(u_{0}\right)$ with nonzero veloc- ity. Then the trajectory falls in the basin of the right minimum of the potential, performing a damped oscillation about it before reaching $\left.z_{3}(F / A), 0\right)$. Once $u_{0}(t)$ has reached a neighborhood of the second spiral point, $u_{-1}(t)$ takes its place and performes a similar motion. The resulting trajectory is depicted in Fig. 20(b). The solution of the complete system (2) is shown in Fig. 21 The wave front velocity is approximately given by the reciprocal of the time $u_{0}(t)$ takes to jump from $z_{1}(F / A)$ to $z_{3}(F / A)$. Notice that the oscillations about $z_{3}(F / A)$ persist a longer time as $\alpha$ decreases and may have finite amplitude. Then we cannot approximate sufficiently well $u_{1}(t)$ by the constant value $U_{3}(F / A)$ for small values of the friction and the one active point approximation breaks down. This explains the discrepancies in Fig. [19] for small $\alpha$. Since the difference $\left[U_{3}(F / A)-U_{1}(F / A)\right]$ is larger for the FK than for the cubic nonlinearity, the approximation $u_{1}(t) \approx U_{3}(F / A)$ is better for the FK nonlinearity as shown in Fig. 19]

\section{DISCUSSION}

We have studied wave front solutions in chains of nonlinear oscillators with inertia and damping. Two analytical methods have been used to construct the wave fronts and their velocities. For piecewise linear models, exact formulas can be found for the wave fronts and the relation between their velocity and the applied stress $F$ as Atkinson and Cabrera did already in 1965 for the conservative case [17]. Different from these authors, we 

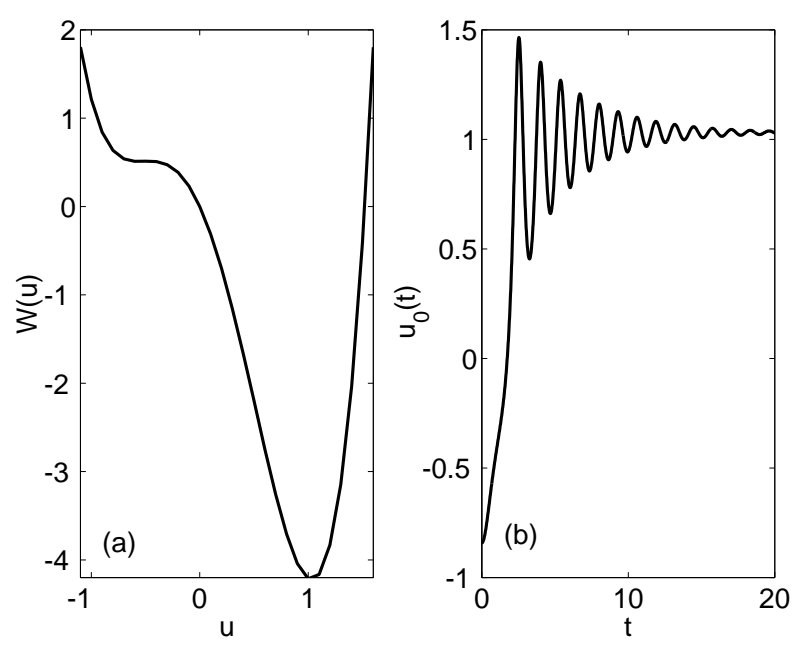

FIG. 20: (a) Potential energy $W(u)$ corresponding to the cubic nonlinearity $h(u)=2 u_{0}+A g\left(u_{0}\right)-F-U_{1}(F / A)-$ $U_{3}(F / A)$, with $A=10$ and $F=2.45$. (b) Trajectories of Eq. (17) with initial condition $\left(U_{1}(F / A), 0\right)$, joining $\left(z_{1}(F / A), 0\right)$ and $\left(z_{3}(F / A), 0\right)$ for $A=10, \alpha=0.5$ and $F=2.45$.

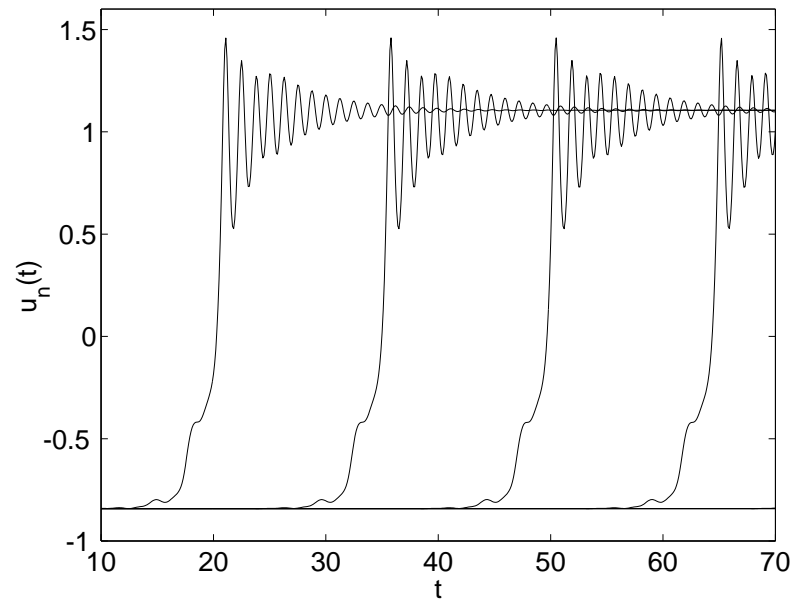

FIG. 21: Numerical solutions $u_{n}(t)$ of Eq. (2) with the cubic nonlinearity for $A=10, \alpha=0.5$ and $F=5.9$.

have also studied the cases of finite and infinite damping. We have depicted the resulting wave front profiles for all damping values and found that they may have oscillatory tails. For zero damping, these tails oscillate with non- decaying amplitude as $n \rightarrow \infty$, which means that a new definition of wave front is needed 23]. We have shown numerically that non-monotone wave fronts with oscillatory tails (wavy wave fronts) may be stable for certain intervals of applied stresses. We have found stable moving wave fronts for $|F|>F_{c d}>0$ (the dynamic Peierls stress), and these fronts coexist with stable static wave fronts for an interval $F_{c d}<|F|<F_{c s}$ ( $F_{c s}$ is the static Peierls stress; static wave fronts exist for $0 \leq|F|<F_{c s}$ ).

We have also conjectured that the global bifurcation diagram for wave front depinning in the presence of inertia and damping is generically as in Fig. 12(b). Then there are infinitely many saddle-node bifurcations between wave front branches in the interval $F_{c d}<|F|<$ $F_{c s}$, accumulating at $c=0$ and $F=F_{c s}$, with $F_{c d}>0$. The basis for this conjecture are our results for the piecewise linear model, that we think are generic also for models with smooth nonlinearities given our numerical results for them. The function $F(c)$ in the piecewise linear model has infinitely many vertical asymptotes (resonances) for zero damping that accumulate as $c \rightarrow 0$ and (we suppose) $F \rightarrow F_{c s}$. These resonances become local maxima of $F(c)$ as a small damping is added to the model. Maxima and minima of $F(c)$ are limit points (saddle-node bifurcations) between branches of wave front solutions in the diagram of Fig. 12(b).

In the literature [19, 21], wave fronts with monotone profiles have been constructed for undamped models. For example, Schmidt 19] showed that $w(x)=\tanh x, x=$ 
$n+t / 2$, is a wave front solution for Eq. (2) with $\alpha=$ $F=0, A=1$ and a potential $V(u)=-3 u^{2} / 4-u^{4} / 8-$ $(\sinh 1)^{-2} \ln \left(1-u^{2} \tanh ^{2} 1\right)$, with $g(u)=V^{\prime}(u)$. For this model, $F_{c s}>0$ 21]. Solving numerically this model for $F \neq 0$, wave fronts with one oscillatory tail on $x>0$ are found for $F>0$, similar to those in Fig. 1(b). For $F<0$, wave fronts with one oscillatory tail on $x<0$ are found instead; see Fig. 1(c). Both types of fronts have $|c|>1 / 2$. This shows that wavy wave fronts are generic and that Schmidt's monotone wave front is a non-generic limiting case separating branches of wavy wave fronts and corresponding to $F_{c d}=0$. A possible bifurcation diagram $|c|$ vs. $F$ for this example could be that in Fig. 12(b) with $F_{c s}>0, F_{c d}=0$ and $\left|c_{m}\right|=1 / 2$ : numerical solution of the model seems to be consistent with a bifurcation diagram having a limit point at $F=0,|c|=1 / 2$, and with reflection symmetry with respect to the $|c|$ axis $(|c|$ in the diagram is even in $F$ ).

\section{Acknowledgments}

This work has been supported by the Spanish MCyT through grants BFM2002-04127-C02, by the Third Regional Research Program of the Autonomous Region of Madrid (Strategic Groups Action), and by the European Union under grant HPRN-CT-2002-00282.

\section{APPENDIX A: NONLINEAR MODEL HAVING A EXPLICIT WAVY WAVE FRONT PROFILE}

It is fairly easy to find smooth $g(u)$ having wave front solutions with undamped oscillatory tails provided their dynamics is conservative. Let us choose a profile of the form:

$$
w(x)= \begin{cases}-1+k_{1} e^{a x} & x \leq 0 \\ 1+k_{2} \cos (b x+c) & x \geq 0\end{cases}
$$

This profile is continuous if $-1+k_{1}=1+k_{2} \cos c$, differentiable if $k_{1} a=-k_{2} b \sin d$ and twice differentiable if $k_{1} a^{2}=-k_{2} b^{2} \cos c$. We set $c=\tan ^{-1}(b / a)$, $k_{1}=2 /\left(1+a^{2} / b^{2}\right)$ and $k_{2}=-k_{1} a /(b \sin c)$. The only restriction on $a$ and $b$ is $b+\tan ^{-1}(b / a)<\pi / 2, a, b>0$ to ensure that $\cos (b x+c)$ is monotone in $0<x<1$. The profile $w(x)$ satisfies:

$$
\begin{gathered}
w_{x x}=\left\{\begin{array}{l}
a^{2}(w+1) \quad \text { for } \quad w<k_{1}-1, \\
-b^{2}(w+1) \quad \text { for } w>k_{1}-1,
\end{array}\right. \\
w(x+1)-2 w(x)+w(x-1)=f(w(x)),
\end{gathered}
$$

with:

$f(w)= \begin{cases}2(w+1)(\cosh a-1) & w<w_{1} \\ (w+1) e^{-a}+Y-2 w & \left(w_{1}, w_{2}\right) \\ k_{2} \cos (W+b)+k_{1} e^{\frac{a}{b}(W-b-c)}-2 w & \left(w_{2}, w_{3}\right) \\ 2(w-1)(\cos b-1) & w>w_{3}\end{cases}$

Here, $W=k_{2}^{-1} \cos ^{-1}(w-1), Y=k_{2} \cos \left\{b a^{-1} \ln [(w+\right.$ 1) $\left.\left./ k_{1}\right]+b+c\right\}, w_{1}=e^{-a} k_{1}-1, w_{2}=k_{1}-1$, and $w_{3}=$ $k_{2} \cos (b+c)-1$. The continuous function $f(w)$ is convex but, for a certain interval of values of $c^{2}$, the function $g_{c}(u)=c^{2} u_{x x}-f(u)$ has three zeroes and is strongly 
asymmetric. For these values of $c$, the system $d^{2} u_{n} / d t^{2}=\quad u_{n}(t)=w(n-c t)$.

$u_{n+1}-2 u_{n}+u_{n-1}-g_{c}\left(u_{n}\right)$ has a traveling wave solution

[*] E-address ana_carpio@mat.ucm.es.

[**] E-address bonilla@ing.uc3m.es.

[1] J. Frenkel and T. Kontorova, J. Phys. USSR, 13, 1 (1938). O.M. Braun and Yu.S. Kivshar, Phys. Rep. 306, $1(1998)$.

[2] F.R.N. Nabarro, Theory of Crystal Dislocations (Oxford University Press, Oxford, UK, 1967).

[3] L.I. Slepyan, Sov. Phys. Dokl. 26, 538 (1981).

[4] P. M. Chaikin and T. C. Lubensky, Principles of condensed matter physics (Cambridge University Press, Cambridge, 1995). Chapter 10.

[5] A. Carpio, L.L. Bonilla and G. Dell'Acqua, Phys. Rev. E, 64, 036204 (2001).

[6] L.L. Bonilla, J. Phys. Condensed Matter 14, R341 (2002).

[7] A.R.A. Anderson and B.D. Sleeman, Int. J. Bif. Chaos, 5, 63 (1995). A. Carpio and L.L. Bonilla, SIAM J. Appl. Math. 63, 619 (2002).

[8] J.P. Keener and J. Sneyd, Mathematical Physiology (Springer, New York, 1998). Chapter 9.

[9] D.R. Nelson, Defects and Geometry in Condensed Matter Physics (Cambridge U.P., Cambridge, UK, 2002).

[10] C. Rebbi and J. Soliani (eds.), Solitons and Particles, (World Sci., Singapore, 1984).

[11] A. Carpio and L.L. Bonilla, Phys. Rev. Lett. 86, 6034 (2001) and SIAM J. Appl. Math. 63, 1056 (2003).

[12] R. Hobart, J. Appl. Phys. 36, 1948 (1965).

[13] V.L. Indenbom, Soviet Phys. - Crystallogr. 3, 193 (1959)
[Kristallografiya 3, 197 (1958)].

[14] J. W. Cahn, Acta Metallurgica 8, 554 (1960).

[15] J.R. King and S.J. Chapman, Eur. J. Appl. Math. 12, 433 (2001).

[16] G. Fáth, Physica D, 116, 176 (1998).

[17] W. Atkinson and N. Cabrera, Phys. Rev. 138, A763 (1965).

[18] J. H. Weiner, Phys. Rev. 136, A863 (1964).

[19] V.H. Schmidt, Phys. Rev. B 20, 4397 (1979).

[20] P.C. Bressloff and G. Rowlands, Physica D 106, 255 (1997).

[21] S. Flach, Y. Zolotaryuk and K. Kladko, Phys. Rev. E 59, 6105 (1999).

[22] M. Peyrard and M.D. Kruskal, Physica D 14, 88 (1984).

[23] In the conservative limit $m \rightarrow \infty$, the oscillations at one of both tails of the wave front do not decay as $n \rightarrow \pm \infty$. This means that a slightly more general definition of wave front solution is needed in the conservative case: $u_{n}(\tau)=w(n-c \tau)$ joins the neighborhoods of $U_{1}(F / A)$ and $U_{3}(F / A)$ (or viceversa) as $n$ increases from $-\infty$ to $\infty$. An infinitesimal amount of damping causes the oscillatory tails of the wave front to decay to either $U_{1}(F / A)$ or $U_{3}(F / A)$ as $n \rightarrow \pm \infty$.

[24] We would like to point out that the numerical solution of Eq. (2) should be calculated by using a scheme of sufficiently high order and small tolerance for the cases $\alpha=0$ or $\alpha>0$ small (typically a standard Rung-KuttaFehlberg scheme of orders $4 / 5$ with tolerance $10^{-5}$ would 
do). Schemes with lower order or larger tolerances have been found to produce spurious traveling waves. For the purpose of checking the appropriatedness of a given numerical scheme, the exact solutions we have discussed are invaluable.

[25] G. Iooss and D.D. Joseph, Elementary stability and bi- furcation theory (Springer, New York, 1980).

[26] I.S. Gradshteyn and I.M. Ryzhik, Table of integrals, series, and products (Academic Press, New York, 1980). Chapter 8. 